\title{
Quantitative Analysis and Effect of SDF, APF, NaF on Demineralized Human Primary Enamel Using SEM, XRD,
} and FTIR

\author{
Zohra Jabin $^{1}$, Iffat Nasim² ${ }^{2}$ Vishnu Priya V ${ }^{3}$, Nidhi Agarwal ${ }^{4}$
}

\begin{abstract}
Introduction: Topical fluoride therapy has proven benefits in the prevention of demineralization. Tooth enamel has shown a great potential for remineralization with an application of topical fluorides if administered at an appropriate time. In an effort to find an effective remineralizing agent, a novel fluoride agent silver diamine fluoride (SDF) has emerged as a potent caries arresting as well as caries preventing agent.

Aim and objective: The present study was aimed at determining the primary tooth enamel resistance to demineralization after topical application of three fluoride agents SDF, APF, and NaF.

Materials and methods: Enamel specimens were prepared from 40 caries-free primary molars. These specimens were randomly allocated into three groups of 10 specimens each and they were treated by different topical fluorides namely: Group I-SDF, group II-Acidulated phosphate fluoride (APF), group III-Sodium fluoride. Three enamel specimens from each group were placed on custom-made acrylic blocks with $5 \times 5$ $\mathrm{mm}$ of an exposed window for scanning electron microscope (SEM) evaluation and rest of the specimens were ground into a fine powder for X-ray diffraction (XRD) and Fourier transform infrared spectroscopy (FTIR) analysis. The tooth blocks and treated samples were subjected to the demineralization process for 168 hours. They were then qualitatively assessed to evaluate their resistance to demineralization using SEM, XRD, and FTIR.
\end{abstract}

Results and conclusion: Morphologically, the enamel of all groups specimens was mostly smooth with some groves and microporosities. Chemically, the $\mathrm{Ca} / \mathrm{P}$ molar ratios of all groups were similar with slight variations. Structurally, the crystalline phases found in enamel by powder XRD were hydroxyapatite and carbonate apatite; and there was a higher amount of incorporated type B carbonate than type A carbonate as evidenced by FTIR. The study concludes that topical application of a 38\% SDF solution can inhibit demineralization of enamel.

Keywords: Primary teeth, Silver diamine fluoride, Sodium fluoride, Tooth remineralization.

International Journal of Clinical Pediatric Dentistry (2021): 10.5005/jp-journals-10005-1988

\section{INTRODUCTION}

Early childhood caries (ECC) is increasing rapidly, despite several measures taken for their decline.

Current concepts in dentistry advocate that more focus should be laid upon the prevention of caries rather than treating or restoring them. This necessitates the use of methods and interventions which prevent tooth demineralization and halts the caries process at its initial level. ${ }^{1-4}$

Under normal oral conditions when the $\mathrm{pH}$ is favorable, the ongoing demineralization and remineralization processes maintain a healthy ecological balance. Saliva promotes remineralization by exerting its buffering action and aids in the continuous uptake of calcium and phosphate ions by the teeth. However, several factors like changes in individual dietary patterns and compromised oral hygiene may bring about a fall in $\mathrm{pH}$ and precipitates demineralization. $^{5}$

If demineralization exceeds the self-regulated remineralization, excessive loss of mineral content may take place, endangering the integrity of the tooth It implies that for preventing the caries process the modulation of the demineralization-remineralization balance is imperative. ${ }^{6}$

Remineralization by topical fluorides can be achieved by incorporating minerals into the enamel. Topical application of fluoride agents forms a thick layer of calcium fluoride on the teeth. Whenever demineralization supersedes remineralization the minerals from this layer are dissolved and enamel remains unaffected. ${ }^{6,7}$

\footnotetext{
1,4 Department of Pedodontics and Preventive Dentistry, Institute of Dental Studies and Technologies, Ghaziabad, Uttar Pradesh, India

${ }^{2}$ Department of Conservative Dentistry and Endodontics, Saveetha Dental College, Saveetha University, Chennai, Tamil Nadu, India

${ }^{3}$ Department of Biochemistry, Saveetha Dental College, Saveetha University, Chennai, Tamil Nadu, India
}

Corresponding Author: Zohra Jabin, Department of Pedodontics and Preventive Dentistry, Institute of Dental Studies and Technologies, Ghaziabad, Uttar Pradesh, India, Phone: +91 9871933858, e-mail: drzohrajabin@gmail.com

How to cite this article: Jabin Z, Nasim I, Vishnu Priya V, et al. Quantitative Analysis and Effect of SDF, APF, NaF on Demineralized Human Primary Enamel Using SEM, XRD, and FTIR. Int J Clin Pediatr Dent 2021;14(4):537-541

Source of support: Nil

Conflict of interest: None

Although topical fluorides contribute largely to the prevention of tooth decay, their preventive action is attributed to their constant availability in the oral cavity which allows them to act directly on the demineralization/remineralization phenomena. ${ }^{8}$

Fluoride varnish is the most preferred method of fluoride delivery but its effectiveness is partial as reported by several researchers. Also, few reported patient compliance issues with its

() The Author(s). 2021 Open Access This article is distributed under the terms of the Creative Commons Attribution 4.0 International License (https://creativecommons. org/licenses/by-nc/4.0/), which permits unrestricted use, distribution, and non-commercial reproduction in any medium, provided you give appropriate credit to the original author(s) and the source, provide a link to the Creative Commons license, and indicate if changes were made. The Creative Commons Public Domain Dedication waiver (http://creativecommons.org/publicdomain/zero/1.0/) applies to the data made available in this article, unless otherwise stated. 
use owing to the unpalatable taste and need for multiple frequent visits for the application cycle. ${ }^{9}$

Silver diamine fluoride (SDF) is a novel fluoride agent and its main constituents are silver, fluoride, and ammonia. The fluoride is present in a high amount which ensures excellent tooth remineralization and silver potentiates its antimicrobial effect. Also, ammonia stabilizes its concentration in the solution for extended periods of time. It has a high fluoride content, cariostatic effect, and renders great ease of application. Its unique properties make it a promising agent for the prevention of caries. ${ }^{10}$

Caries arresting effect of SDF is well documented in the available literature since most of the studies done so far on SDF were aimed at evaluating its effect on active caries. However, the literature exploring its preventive effect after application on sound teeth is still scarce. Thus, it is of interest to conduct a qualitative assessment of primary tooth enamel after the application of SDF and different topical fluorides.

$X$-ray diffraction (XRD) is a technique for characterizing crystalline materials and is used to evaluate the crystal structure of the samples. It provides information on crystal structure, chemical phases, preferred crystal orientation (texture), and other structural parameters. Fourier transform infrared spectroscopy (FTIR) analyzes the enamel crystallinity by measuring molecular bands at different vibration modes. This technique provides an insight into the crystal sizes and order of arrangement within the hydroxyapatite crystals. SEM displays the morphological characteristics of enamel for its qualitative assessment.

The purpose of this study was to assess primary tooth enamel resistance to demineralization after topical SDF application. This study was performed in vitro comparing SDF with two other topical fluorides (NaF and APF) having different fluoride concentrations, by the qualitative assessment of primary tooth enamel using SEM, $\mathrm{XRD}$, and FTIR.

\section{Materials and Methods}

Sound primary teeth that were freshly extracted due to preshedding mobility or recently exfoliated were obtained from the department. Teeth showing the presence of caries, teeth with fluorosis, or other developmental defects, fractures, or restorations were not included in the study. All teeth were immersed in sodium hypochlorite solution overnight and were gently cleaned in the morning to remove soft tissue debris using a soft bristle brush. After which teeth were stored at $4^{\circ} \mathrm{C}$ in $0.2 \%$ thymol solution before the initiation of the study. Before the intervention, each tooth was rinsed with deionized water.

The crown of the selected teeth was separated from the root and enamel was gently sliced from the coronal part. These thin enamel slices received topical fluorides.

\section{Group Division and Agent Application}

Enamel slices were divided into three groups randomly. Each group (Group I-SDF, group II-APF, group III-NaF) comprised 10 samples, which were treated by topical fluorides agents. Each sample was painted with an assigned agent as per the manufacturer's instruction. The samples were dried for 30 minutes to activate the agents.

\section{Specimen Preparation}

Tooth blocks were made from cold cure acrylic by pouring it into preformed molds. Out of total samples, three enamel slices from each group were randomly selected for SEM evaluation. These enamel slices were embedded on the top surface of cylindrical tooth blocks. They were coated by nail varnish all over the surface except for an exposed small window $(5 \times 5 \mathrm{~mm})$ left in the middle part of the sample.

\section{Preparation of Demineralizing Solution}

The demineralization solution used in this study contained $2.2 \mathrm{mM}$ $\mathrm{CaCl}_{2} .2 \mathrm{mM} \mathrm{NaHPO}$, and $50 \mathrm{mM}$ acetic acid. The $\mathrm{pH}$ was maintained at 4.4 by adding $1 \mathrm{M} \mathrm{KOH}$.

\section{Demineralization}

Demineralization solution $(60 \mathrm{~mL})$ was poured into three beakers and each group sample was immersed in them individually. The demineralization process was done for 7 days during which the beakers were kept on a vibrator to ensure continuous shaking of samples. At the end of the 7th day, all blocks and slices were taken out and thoroughly dried.

\section{SEM Evaluation}

Scanning electron microscope images were obtained at $600 \times$ and $1,200 \times$ for visualizing the morphological characteristics of each group sample.

\section{XRD and FTIR Analysis}

Enamel slices were milled into fine powder. Half of this powder was taken for XRD analysis by means of a diffractometer. ${ }^{11}$ The rest half was used for FTIR analysis. The diffraction intensity as a function of the angle 2-theta was measured between $20^{\circ}$ and $90^{\circ}$.

\section{FTIR}

Fourier transform infrared spectroscopy spectra of enamel powder were recorded, before which the enamel powder was mixed with $\mathrm{KBr}$ in an agate mortar. Fourier Transform Infrared Spectroscopy measured the absorbance of carbonate and phosphate bands.

\section{Results}

\section{SEM}

SEM images of all samples showed hemogeneously arranged crystals with well-coalesced enamel rods without any loss of structural characteristics. Enamel was covered with a $\mathrm{CaF}_{2}$ layer which showed a granular morphology. Silver diamine fluoride treated enamel samples exhibited a similar appearance with the presence of honeycomb structure and hydroxyapatite prisms (Fig. 1A).

APF-treated sample (Fig. 1B) showed a remineralized layer of enamel at the surface and also along the prismatic borders. Few NaF-treated samples showed exposed prisms with irregular shapes (Fig. 1C). Although the morphological pattern of most samples was smooth with some grooves and microporosities. The treatments with all three topical fluorides significantly inhibited enamel demineralization by forming $\mathrm{CaF}_{2}$ and fluoride-containing appetites at the enamel surface, however, enamel rod characteristics could not be appreciated in APF samples.

\section{XRD}

The diffractograms shown in graphs $2 A, B$ and $C$ (Fig. 2) indicate that there are no crystallographic changes of statistical significance observed in enamel when pretreated with three different topical 

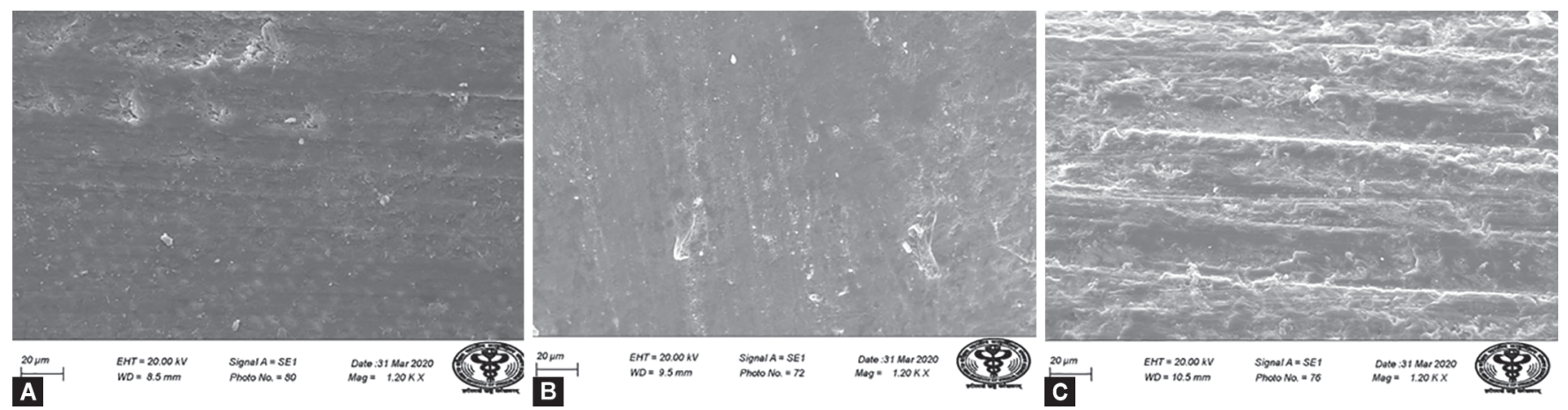

Figs 1 A to C: SEM images of SDF, APF and NaF samples
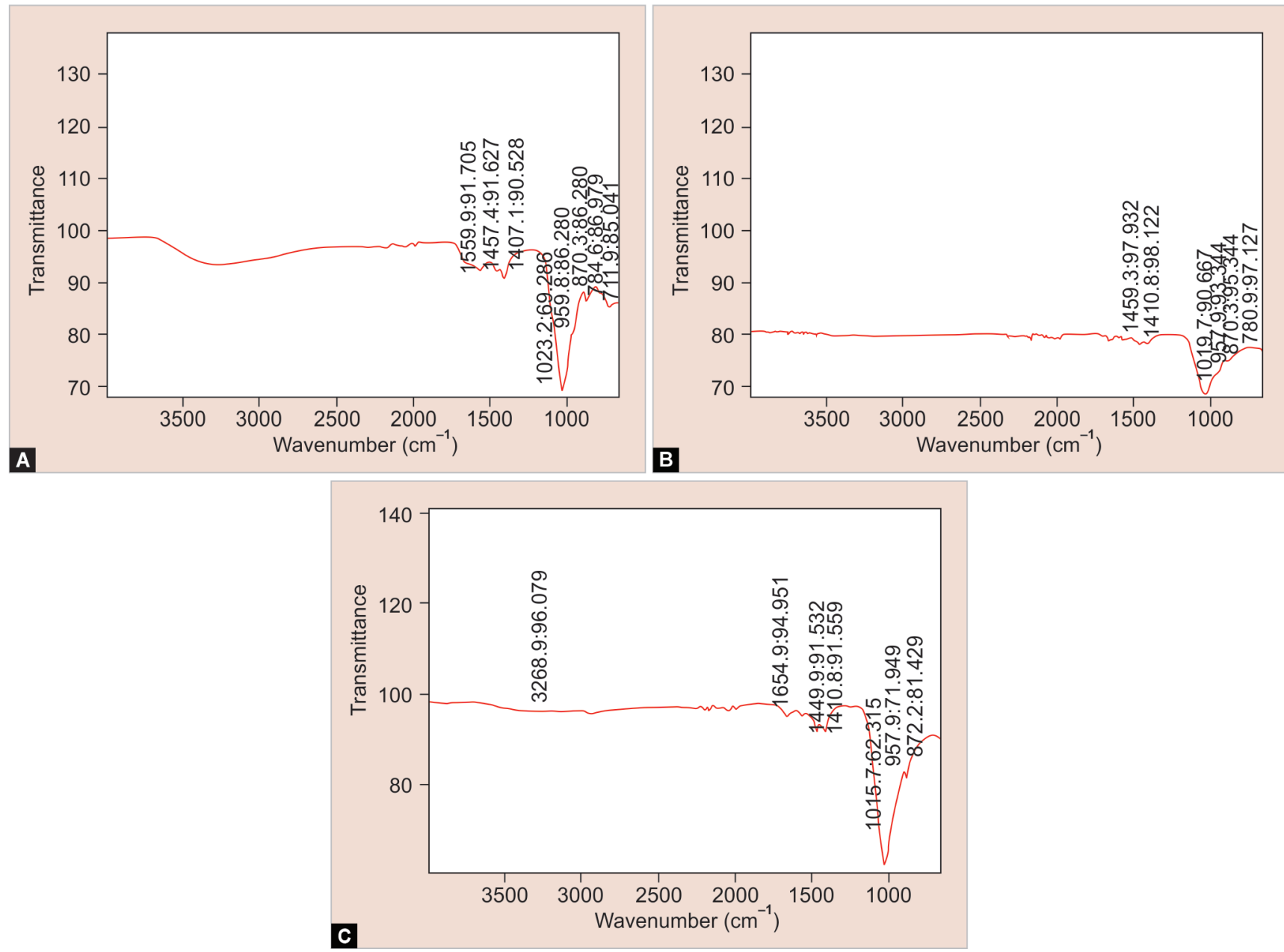

Figs $2 \mathrm{~A}$ to C: FTIR graphs of SDF, APF and NaF samples

fluorides. The positions of all diffraction peaks were largely similar to each other. As the crystallites of human tooth enamel have a "prism" like shape, their size is reported to vary depending on the peak intensities. On observing the intensity of peaks in XRD patterns, several phases of enamel-like calcium silicate, potassium calcium phosphate, and hydrated calcium phosphate were discovered. In NaF pretreated samples, the initial peaks were observed at $2 \theta$ values of $26.14^{\circ}$ and $31.16^{\circ}$. These peaks are related to crystalline and amorphous organic phases of primary tooth enamel. $2 \theta$ values of $36.13,40.20^{\circ}$, and $46.92^{\circ}$ were also obtained.
APF pretreated enamel showed the initial peaks at $2 \theta$ values of $20.13^{\circ}$ and $28.22^{\circ}$ along with $2 \theta$ values of $30.12,35.18^{\circ}, 41.10^{\circ}$, 46.07, and 50.10.

Silver diamine fluoride pretreated enamel showed the initial peaks at $2 \theta$ values of $12.98^{\circ}, 26.14,32.53,37.22,41.57$, and $48.96^{\circ}$ which were suggestive of enamel organic phases. $2 \theta$ values of 30.12 , $35.18^{\circ}, 41.10^{\circ}, 46.07$, and 50.10 were also obtained.

\section{FTIR}

Fourier Transform Infrared Spectroscopy registered the tissue spectra as between 4,000 and $400 \mathrm{~cm}^{-1}$. Fourier Transform Infrared 
Spectroscopy analysis of powdered enamel samples was done to characterize the enamel and check the possible functional groups present after pretreatment with different topical fluorides. Fourier Transform Infrared Spectroscopy spectra show various functional groups found in the enamel powder treated with Naf, APF, and SDF as seen in Figure 3. For sodium fluoride, the broad peak at $3,268 \mathrm{~cm}^{-1}$ corresponds to $\mathrm{O}-\mathrm{H}$ stretching vibrations of the hydroxyl groups. The amide I vibration arising from $\mathrm{C} \hat{\mathrm{mO}}$ stretching was centered in the $1,654 \mathrm{~cm}^{-1}$ band. The peak at $1,559 \mathrm{~cm}^{-1}$ is also due to amide $\mathrm{C} \hat{\mathrm{mO}}$ stretching vibration. The characteristic bands for the alkanes functional group $(\mathrm{H}-\mathrm{C}-\mathrm{H})$ were observed at 1,449 and $1,410 \mathrm{~cm}^{-1}$. The peak at $1,015 \mathrm{~cm}^{-1}$ attributes to $\mathrm{C}-\mathrm{O}$ [carbonyl group] stretching vibration. Regarding the phosphates, bands could be localized at $957 \mathrm{~cm}^{-1}$ The major of carbonate was located at $872 \mathrm{~cm}^{-1}$.

For APF, fluoride bands were visible in the range 500 to $2,000 \mathrm{~cm}^{-1}$, the FTIR spectra showed the characteristic bands of carbonate at $1,019 \mathrm{~cm}^{-1}$, alkanes $(\mathrm{H}-\mathrm{C}-\mathrm{H})$ at 1,410 , and the peak of $870 \mathrm{~cm}^{-1}$ attributes to $\mathrm{C}-\mathrm{H}$ bending vibration. Silver diamine fluoride applied powdered enamel samples show a peak at 1,653 which denotes amide stretching. The band at $1,457 \mathrm{~cm}^{-1}$ corresponds to the $\mathrm{H}-\mathrm{C}-\mathrm{H}$ band for the alkanes functional group. The $\mathrm{C}-\mathrm{O}$ carbonyl region shows stretching vibration at $1,023 \mathrm{~cm}^{-1}$, regarding the phosphates peak, was localized at $959 \mathrm{~cm}^{-1}$ in SDFpowdered samples.

\section{Discussion}

Modern dentistry emphasizes that early detection and prevention of tooth demineralization should be done so that the need for an invasive restorative approach does not arise. The 38\% SDF has potent antimicrobial properties and it helps in remineralization due to its high fluoride content (44,800 ppm), moreover, its ease of application and noninvasive approach makes it a promising topical fluoride agent. $^{12}$

The present study was conducted on the primary tooth enamel. Although ample research has been done to evaluate the remineralization characteristics of permanent tooth enamel less attention was given to analyze the same for their primary counterparts. Derise et al. found primary and permanent teeth differ in their, concentration and percentage of $\mathrm{Ca}$ and $\mathrm{P} .{ }^{13}$ Since primary and permanent teeth bear startling differences between the micropatterns and structural morphology, it is imperative to study both of them individually. The present study explored qualitative changes occurring in sound enamel, post SDF, and other topical fluoride applications and assessed their demineralization inhibiting action by means of morphological characterization. SEM, structural
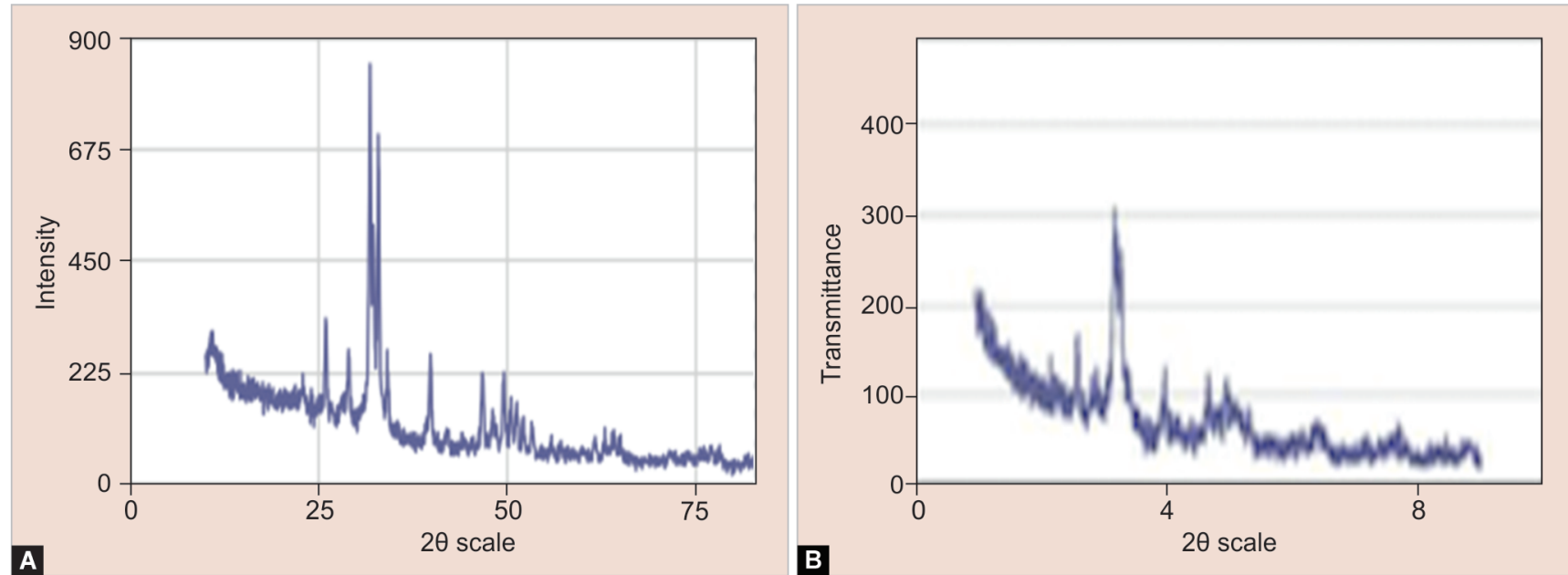

A

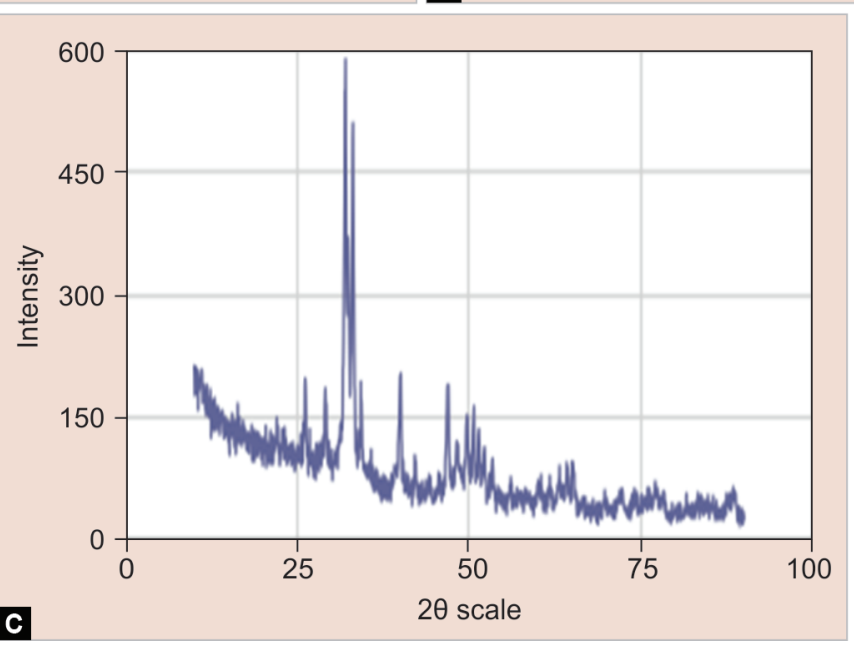

Figs $3 A$ to C: XRD graphs of SDF, APF and NaF samples 
characterization. X-ray diffraction and infrared microscopy spectral analysis (FTIR).

Resistance against demineralization in a given sample is measured by estimation of its crystallinity index. This gives an idea about crystal size and order. ${ }^{14}$

$\mathrm{X}$-ray diffraction and FTIR techniques represent the efficient and promising qualitative methods for analyzing the crystalline phases of enamel, and it is emphasized that both methods be used in synergy.

Fourier Transform Infrared Spectroscopy results of the present study showed high, sharp peaks of carbonate, phosphate, and amide I, II, and/or III bands. This effect is thought to be due to remineralizing effects of topical fluorides on primary tooth enamel by their incorporation into the crystal lattice.

The SEM observations showed that the enamel is characterized by both amorphous and prismatic hydroxyapatite. The exposure to demineralizing solution rendered the prisms exposed resulted in deprived interprismatic and prismatic substances in all groups.

The application of topical fluorides before demineralization for 4 minutes prompts hemogeneous consistent remineralization of enamel by forming a rich layer of carbonate-hydroxyapatite coating which covers the enamel structure and provided protection against demineralization. In the present study, the resistance to demineralization was similar in all three groups.

Several studies researched the remineralizing effect of SDF by using an alternative method of microhardness measurement of carious lesions treated with SDF and observed an approximate depth of $150 \mu \mathrm{m} .{ }^{15}$

The XRD patterns, SEM images, and FTIR spectra display an enamel repair process resulted due to the application of topical fluorides before enamel demineralization.

Available literature suggests that caries can be efficiently treated by direct application of SDF. ${ }^{16,17}$ The calcium, phosphate, and fluoride ions knocked out from the teeth due to $\mathrm{pH}$ changes and ecological imbalance can be reabsorbed resulting in highly remineralized and acid-resistant fluorapatite crystals. Thus, SDF application may be considered an innovative approach to remineralization and subsequent caries prevention.

Although the present study yields useful results, its potential limitation is that the evaluation was done in vitro conditions. As the in vitro conditions does not simulate dynamic complex biological oral system further in vivo clinical trials needs to be conducted to gain more valuable information regarding demineralization inhibiting the potential of SDF.

\section{References}

1. West NX, Maxwell A, Hughes JA, et al. A method to measure clinical erosion: the effect of orange juice consumption on erosion of enamel. J Dentis 1998;26(4):329-335. DOI: 10.1016/s0300-5712(97) 00025-0.

2. West NX, Hughes JA, Parker DM, et al. Development and evaluation of a low erosive blackcurrant juice drink. 2. Comparison with a conventional blackcurrant juice drink and orange juice. J Dentis 1999;27(5):341-344. DOI: 10.1016/s0300-5712(98)00070-0.

3. Hughes JA, West NX, Parker DM, et al. Development and evaluation of a low erosive blackcurrant juice drink in vitro and in situ. 1. Comparison with orange juice. J Dentis 1999;27(4):285-289. DOI: 10.1016/s0300-5712(98)00069-4.

4. Hughes JA, West NX, Parker DM, et al. Development and evaluation of a low erosive blackcurrant juice drink 3 . Final drink and concentrate, formulae comparisons in situ and overview of the concept. J Dentis 1999;27(5):345-350. DOI: 10.1016/s0300-5712(98)00068-2.

5. Soares R, De Ataide IN, Fernandes M, et al. Assessment of enamel remineralisation after treatment with four different remineralising agents: a scanning electron microscopy (SEM) study. J Clin Diagn Res 2017;11(4):ZC136-ZC141. DOI: 10.7860/JCDR/2017/23594.9758.

6. Prabhakar AR, Jaiswal Manojkumar A, Basappa N. Comparison of the remineralizing effects of sodium fluoride and bioactive glass using bioerodible gel systems. J Dent Res Dent Clin Dent Prospects 2009;3:117-121.

7. Ganss C, Klimek J, Giese K. Dental erosion in children and adolescents - a cross-sectional and longitudinal investigation using study models. Community Dentis Oral Epidemiol 2001;29(4):264-271. DOI: 10.1034/j.1600-0528.2001.290405.x.

8. Takagi S, Liao H, Chow LC. Effect of tooth-bound fluoride on enamel demineralization/remineralization in vitro. Caries Res 2000;34(4):281288. DOI: $10.1159 / 000016603$.

9. Demito CF, Vivaldi-Rodrigues $\mathrm{G}$, Ramos AL, et al. The efficacy of a fluoride varnish in reducing enamel demineralization adjacent to orthodontic brackets: an in vitro study. Orthodont Craniofac Res 2004;7(4):205-210. DOI: 10.1111/j.1601-6343.2004.00305.x.

10. Jabin Z, Vishnupriya V, Agrawal N, et al. Effect of $38 \%$ silver diamine fluoride on control of dental caries in primary dentition: a systematic review. J Fam Med Prim Care 2020;9(3):1302. DOI: 10.4103/jfmpc. jfmpc_1017_19.

11. Behroozibaksh M, Hajizamani H, Shekofteh K, et al. Comparative assessment of the crystalline structures of powder and bulk human dental enamel by X-ray diffraction analysis. J Oral Biosci 2019;61(3):173-178. DOI: 10.1016/j.job.2019.06.003.

12. Chu CH, Lo EC, Lin HC. Effectiveness of silver diamine fluoride and sodium fluoride varnish in arresting dentin caries in Chinese preschool children. J Dent Res 2002;81(11):767-770. DOI: 10.1177/0810767.

13. Derise NL, Ritchey SJ, Furr AK. Mineral composition of normal human enamel and dentin and the relation of composition to dental caries. I-Macrominerals and comparison of methods of analyses. J Dent Res 1974;53(4):847-852. DOI: 10.1177/00220345740530041501.

14. De Menezes Oliveira MAH, Torres CP, Gomes-Silva JM, et al. Microstructure and mineral composition of dental enamel of permanent and deciduous teeth. Microsco Res Techni 2010;73(5):572577. DOI: 10.1002/jemt.20796.

15. Shetty S, Hegde M, Bopanna T. Enamel remineralization assessment after treatment with three different remineralizing agents using surface microhardness: an in vitro study. J Conserv Dent 2014;17(1):4952. DOI: 10.4103/0972-0707.124136.

16. Yee R, Holmgren C, Mulder J, et al. Efficacy of silver diamine fluoride for arresting caries treatment. J Dent Res 2009;88(7):644-647. DOI: 10.1177/0022034509338671.

17. Llodra JC, Rodriguez A, Ferrer B, et al. Efficacy of silver diamine fluoride for caries reduction in primary teeth and first permanent molars of schoolchildren:36-month clinical trial. J Dent Res 2005;84(8):721-724. DOI: $10.1177 / 154405910508400807$. 